\title{
Role and Effect of Radiation Therapy for Retinoblastoma Treated with Prior Intra-Arterial Chemotherapy
}

\section{Kiran Turaka and Aruna Turaka*}

Department of Radiation Oncology, Paramount Oncology Group, Albert G. and Helen Nassif Radiation Center, Unity Point Health-St. Luke's Hospital, Cedar Rapids, IA, USA

"Corresponding author: Aruna Turaka, Department of Radiation Oncology, Paramount Oncology Group, Helen Nassif Radiation CenterUnity Point Health-St. Luke's Hospita, Cedar Rapids, IA, USA, Tel: 319-861-6944; Fax: 319-861-6945; E-mail: arunaturaka@gopog.com

Received date: Jan 23, 2016; Accepted date: Jan 25, 2016; Publication date: Jan 31, 2016

Copyright: (c) 2016 Turaka A, et al. This is an open-access article distributed under the terms of the Creative Commons Attribution License; which permits unrestricted use; distribution; and reproduction in any medium; provided the original author and source are credited.

Keywords: Chemotherapy; External beam radiation; Fluoroscopy; Plaque radiotherapy

RetinoBlastoma (RB) is the most common intraocular tumor in the world among children. It is one of the life threatening tumors in children in underdeveloped countries. In the developed countries, due to early diagnosis and latest treatment techniques, the mortality is less. Systemic chemotherapy either alone or along with periocular injections with or without Radiation Therapy ((RT) External Beam Radiation (EBRT), plaque radiotherapy, proton beam therapy) and focal therapy (laser photocoagulation, or cryotherapy) has been used to treat the retinoblastoma with good success rates. Second Malignant Neoplasms (SMN) are known to occur after treatment with systemic chemoreduction and EBRT among the children of retinoblastoma especially in hereditary RB than sporadic RB cases $[1,2]$. Therefore, the $\mathrm{RB}$ treatment goal is not only to salvage the globe, preserve vision but also to reduce the radiation exposure to reduce the risk of SMN's.

Recently, intra-arterial chemotherapy (IAC) is being used widely in the treatment of retinoblastoma especially in relapsing-recurring and/or progressive RB [3-6]. This selective chemotherapy delivery method is done by using the agent Melphalan with a dose range of 3-5 mg per dose per eye (depending on age) for 1-5 cycles [5-7]. Other chemotherapy agents used for IAC are 0.3-0.6 mg of Topotecan, and $25-50 \mathrm{mg}$ of Carboplatin [8,9]. This newer treatment method is reported to have to good tumor regression rates, salvages the globe and avoids high-dose of radiation to the treating eye, fellow eye and neighboring structures [5]. Few authors reported complications such as vitreous hemorrhage, cataract, vasculopathy and radiation-related toxicities with IAC [3-7]. The major question to be raised with this new treatment modality is development of distant metastasis as it is a much localized chemotherapy and also $\mathrm{RB}$ is the most common metastasizing tumor among children. In that situation, what concerns the ocular oncologists and the pediatric oncologists is does additional radiation therapy has any role in the treatment of retinoblastoma apart from systemic chemotherapy? Another concern to the oncologists is, do any radiation-related side effects occur (such as SMN in future) from $\mathrm{x}$-ray fluoroscopy during arterial infusion among IAC patients? Few oncologists are using systemic chemotherapy along with intraarterial chemotherapy to prevent the metastatic disease.

EBRT is used in approximately $45 \%$ of the patients after IAC by Thampi and colleagues [5]. None of these patients received prior RT before IAC. EBRT controlled the progressing vitreous seeds and recurrent $\mathrm{RB}$ in this study. Minor complication such as cataract is reported among these limited series patients. None of the patients developed any hematological malignancies or sarcomas with a short available follow-up time (14.5 months). In this study, the dose of EBRT received by the patients was not mentioned [5]. Plaque brachytherapy is used as adjuvant and salvage therapy in $\mathrm{RB}$ patients with recurrence after IAC $[8,9]$. The mean dose delivered via Iodine- 125 plaque was 42 Gy at an average duration of 73 hours. Plaque RT after IAC achieved an ocular survival of approximately $79 \%$ (at 18 months). Tumor recurrence rate was less following plaque RT after IAC with none developing any distant metastasis. Tumor recurrence was seen in patients treated with out-of volume target than in-volume target and also among patients who had plaque placed in horizontal meridian and superior hemisphere than inferior hemisphere. Most of the patients have improved or stable retinal function as measure by the eletroretinogram. Complications noted with plaque RT are cataract, optic neuropathy and vitreoretinopathy. The limitations of this study are lack of long-term radiation effects after plaque RT with prior IAC [9].

X-ray fluoroscopy helps in guiding the catheter during the IAC procedure for accurate delivery of chemotherapy to the ophthalmic artery and is known to cause radiation side-effects $[10,11]$. The amount of radiation exposure following IAC is an average dose $20 \mathrm{mGy}$ per eye per cycle as reported in literature [5]. This low dose could have caused the cataracts among these patients, but none developed SMN [5]. However, few authors reported different radiation exposure doses following IAC. The range of doses to the treated eye is from 5.5 to 191 mGy $[10,11]$. Short-term complications noted are eye lid erythema, madarosis, cataract and chorioretinal atrophy due to infarction. Some authors reported possibility of SMN due to radiation exposure during IAC [10-12].

Radiation therapy following IAC is effective salvage/adjuvant therapy to control the recurrent or progressive $\mathrm{RB}$ and advancing vitreous seeds. RT can cause minor short-term toxicities among these patients. Long-term toxic effects of radiation such second malignancies can occur especially among the hereditary RB cases but no data is currently reported about RT effects following IAC to be the best of our knowledge. Fluoroscopy induced short-term radiation effects following IAC are localized to the eye and not life threatening. There are limited studies reporting the long-term study results of fluoroscopic induced radiation toxicities.

\section{References}

1. Fujiwara T, Fujiwara M, Numoto K, Ogura K, Yoshida A, et al. (2015) Yonemoto T, Suzuki S, Kawai A. Second primary osteosarcomas in patients with retinoblastoma. Jpn J Clin Oncol 45: 1139-1145. 
Citation: Turaka A, Turaka K (2016) Role and Effect of Radiation Therapy for Retinoblastoma Treated with Prior Intra-Arterial Chemotherapy. J Nucl Med Radiat Ther 7: e115. doi:10.4172/2155-9619.1000e115

Page 2 of 2

2. Turaka K, Shields CL, Meadows AT, Leahey A (2012) Second malignan neoplasms following chemoreduction with carboplatin, etoposide, and vincristine in 245 patients with intraocular retinoblastoma. Pediatr Blood Cancer 59: 121-125.

3. Akyüz C, Kırath H, Şen H, Aydın B, Tarlan B, et al. (2015) Varan A. IntraArterial Chemotherapy for Retinoblastoma: A Single-Center Experience. Ophthalmologica 234: 227-232.

4. Francis JH, Abramson DH, Gobin YP, Marr BP, Tendler I, et al. (2015) Brodie SE, Dunkel IJ. Efficacy and toxicity of second-course ophthalmic artery chemosurgery for retinoblastoma. Ophthalmology 122: 1016-1022.

5. Thampi S, Hetts SW, Cooke DL, Stewart PJ, Robbins E, et al. (2013) Superselective intra-arterial melphalan therapy for newly diagnosed and refractory retinoblastoma: results from a single institution. Clin Ophthalmol 7: 981-989.

6. Jabbour P, Chalouhi N, Tjoumakaris S, Gonzalez LF, Dumont AS, et al (2012) Pearls and pitfalls of intraarterial chemotherapy for retinoblastoma. J Neurosurg Pediatr 10: 175-181.

7. Suesskind D, Schrader M, Foerster MH, Ernemann U, Aisenbrey S (2014) Cataract formation: a possible complication of intra-arterial chemotherapy for retinoblastoma. Eur J Ophthalmol 24: 449-453.
8. Marr BP, Brodie SE, Dunkel IJ, Gobin YP, Abramson DH (2012) Threedrug intra-arterial chemotherapy using simultaneous carboplatin, topotecan and melphalan for intraocular retinoblastoma: preliminary results. Br J Ophthalmol 96: 1300-1303.

9. Francis JH, Barker CA, Wolden SL, McCormick B, Segal K, et al. (2013) Salvage/adjuvant brachytherapy after ophthalmic artery chemosurgery for intraocular retinoblastoma. Int J Radiat Oncol Biol Phys 87: 517-523.

10. Gobin YP, Rosenstein LM, Marr BP, Brodie SE, Abramson DH (2012) Radiation exposure during intra-arterial chemotherapy for retinoblastoma. Arch Ophthalmol 130: 403-404.

11. Vijayakrishnan R, Shields CL, Ramasubramanian A, Emrich J, Rosenwasser $\mathrm{R}$, et al. (2010) Irradiation toxic effects during intra-arterial chemotherapy for retinoblastoma: should we be concerned? Arch Ophthalmol 128: 1427-1431.

12. Shields CL, Bianciotto CG, Jabbour P, Griffin GC, Ramasubramanian A, et al. (2011) Intra-arterial chemotherapy for retinoblastoma: report No. 2, treatment complications. Arch Ophthalmol 129: 1407-1415. 